\title{
Relationship Between the Consumption of
} Milk-Based Oils Including Butter and Kermanshah Ghee with Metabolic Syndrome: Ravansar Non-Communicable Disease Cohort Study

This article was published in the following Dove Press journal:

Diabetes, Metabolic Syndrome and Obesity: Targets and Therapy

\author{
Elham Ahmadi' \\ Hadi Abdollahzad (D) 2,3 \\ Yahya Pasdar (D) ${ }^{2}$ \\ Shahab Rezaeian (iD $^{3}$ \\ Jalal Moludi $\mathbb{B}^{3}$ \\ Seyed Mostafa Nachvak ${ }^{2}$ \\ Roghayeh Mostafai $\mathbb{1 D}^{2}$ \\ 'Student Research Committee, \\ Kermanshah University of Medical \\ Sciences, Kermanshah, Iran; ${ }^{2}$ Department \\ of Nutrition Sciences, School of \\ Nutrition Sciences and Food Technology, \\ Kermanshah University of Medical \\ Sciences, Kermanshah, Iran; ${ }^{3}$ Research \\ Center for Environmental Determinants \\ of Health, Health Institute, Kermanshah \\ University of Medical Sciences, \\ Kermanshah, Iran
}

Correspondence: Hadi Abdollahzad Department of Nutrition Sciences, School of Nutrition Sciences and Food Technology, Kermanshah University of Medical Sciences, Isar Sq., Kermanshah P.O. Box 67I985I35I, Iran Email hadi_nut@yhoo.com
Background: The prevalence of metabolic syndrome (MetS) in recent years has been growing in different societies, which may be due to lifestyle changes including changes in diet, in particular the consumption pattern of edible oils. The purpose of this study was to investigate the relationship between the consumption of animal oils including butter and Kermanshah ghee with MetS and its components in the adult population of Ravansar NonCommunicable Disease (RaNCD) cohort study.

Methods: This cross-sectional study was carried out on 5550 adults aged 35-65 years using baseline data of Ravansar's prospective study center in Iran. MetS was defined according to the criteria of modified NCEP ATP III for Iranian adults. Relationship between the consumption of butter and Kermanshah ghee and MetS was analyzed by logistic regression model using STATA software.

Results: In our study, the frequency of MetS was $31.40 \%$. The mean body mass index and mean age were $27.1 \pm 4.6 \mathrm{~kg} / \mathrm{m}^{2}$ and $47.6 \pm 8.2$ years. The mean values of consumed butter and Kermanshah ghee were $3.3 \pm 1.8$ and $5.1 \pm 2.3 \mathrm{~g}$ /day, respectively. After adjusting the confounding variables, the highest to the lowest quintile of butter and Kermanshah ghee consumption showed a reverse correlation with the MetS (OR $=0.7,95 \% \mathrm{CI}=0.5-0.9)$ and $(\mathrm{OR}=0.7,95 \% \mathrm{CI}=0.6-0.9)$, respectively.

Conclusion: This study revealed a reverse relationship between milk and Kermanshah ghee consumption with MetS and its components. Therefore, consumption of milk-based oils may be associated with lower cardiovascular risk factors.

Keywords: butter, Kermanshah ghee, metabolic syndrome, oil, Ravansar cohort

\section{Background}

Metabolic syndrome (MetS) refers to a cluster of metabolic disorders including insulin resistance, hypertension, abdominal obesity, dyslipidemia and systemic inflammation $^{1}$ that increase the risk of non-communicable diseases such as type 2 diabetes (T2D) and cardiovascular diseases (CVD). ${ }^{2}$ According to the National Cholesterol Education Program (NCEP/ATP III) norms, the occurrence of MetS in the world varies from $8 \%$ to $43 \%$ in men and $7 \%$ to $56 \%$ in women ${ }^{3}$ and varies from $47.2 \%$ to $60.0 \%$ in Iran. ${ }^{4}$ The increasing prevalence of MetS can be attributed to changes in lifestyle, especially the diet. ${ }^{5}$ The amount and type of edible oils are among the most vital factors that regulate the health of our diet. ${ }^{6}$ One group of these 
oils is animal-origin oils, in particular dairy fat which contains saturated and trans-fatty acids (TFA). ${ }^{7}$ One study showed that SFAs may impair insulin sensitivity, ${ }^{8}$ however in a clinical study decreasing SFAs in diet did not affect insulin sensitivity in people with MetS. ${ }^{9}$ A recent review regarding the effects of dairy-derived SFAs consumption on the risk of MetS indicated that there is not enough evidence to support current dietary guidelines for reducing all dietary SFAs regardless of the dietary source, food matrix and composition. ${ }^{10}$

Evidence on the health effects of animal oils are inconclusive, their effects largely depend on the amount of saturated and trans fats in these products. While some studies indicate that animal oils increase the risk of cardiovascular diseases because of their high SFAs content, ${ }^{7,11}$ others have reported that trans-fatty acids in dairy fat do not increase the risk of cardiovascular diseases. ${ }^{12}$ The results of several studies point to that using animal oils significantly increases total cholesterol, HDL-C, and very low-density lipoprotein (VLDL) and decreases Apolipoprotein A (Apo-A) level. ${ }^{13,14}$ On the contrary, several studies have shown that consumption of animal oils as much as about $10 \%$ of total fat intake has no adverse effect on the lipid profile. ${ }^{15,16}$ In a large multicenter cohort study, the consumption of high-fat dairy products, butter, and yogurt showed a strong inverse relationship (linear dose-response) with the risk of MetS. ${ }^{17}$ In another study, however, using butter was directly correlated with the incidence of MetS. ${ }^{7}$ Butter and ghee are commonly used in many parts of Iran, including Kermanshah, where traditionally they account for a significant portion of total oil consumed by households. ${ }^{18}$

Despite the prevalent use of butter and ghee in the common diet of Kermanshah people and the inconsistency of the existing reports on the health effects of these products, there has been no study on the relationship between butter and Kermanshah ghee consumption and the risk of MetS in this population. To close this gap in the literature, this study investigated the relationship between the consumption of oils with animal origin including butter and Kermanshah ghee and MetS and its components in the adult population of Ravansar Non-Communicable Disease (RaNCD) cohort study.

\section{Methods}

\section{Study Design}

This cross-sectional study was performed on baseline data of 9951 permanent residents of Ravansar, including men and women aged 35-65 years that started in 2014. Ravansar is a city in Kermanshah province in west of Iran with a population of over 50,000 people, who are almost entirely of Kurdish ethnicity. This study is part of the PERSIAN (Prospective Epidemiological Research Studies of Iran) mega-cohort study. Details of the Ravansar cohort study protocol have been introduced in previous studies. ${ }^{19,20}$

\section{Population}

The study was designed as a census survey of participants in the RaNCD cohort study. Based on the inclusion criteria, 4401 of participants were excluded due to not having an usual daily energy intake (not at the range of 1.35-2.39 EI:BMR ratio) $(n=2172)$, being diagnosed with noncommunicable diseases for more than 1 year $(n=2195)$, the lack of biochemical data $(n=30)$, and the lack of data on oil consumption $(n=4)$. Ultimately, the data of 5550 participants (2986 females and 2564 males) were investigated in the current study.

\section{Data Collection}

Demographic (age, gender, smoking and alcohol use, the history of diseases and medication), anthropometric and biochemical data, blood pressure, nutrition status of participants, and socioeconomic status (SES) including employment status; incomes, availability of electricity, access to safe drinking water and natural gas for heating and cooking, transport coverage; marital status; number and type of marriages (first-degree or second-degree familial marriage or none); spouse's job; region of residency, number of domestic and international trips; access to landline and mobile phones, the internet and the extent of cell phone use were acquired. ${ }^{19,20}$ Physical activity was assessed using the standardized RaNCD cohort physical activity questionnaire, which measures this parameter in metabolic equivalent per day (MET-h/day). ${ }^{20}$ Data were collected by the relevant trained experts. A brief description of other data collection is provided in the following.

\section{Food Intake Assessment}

Food intake was studied using the Ravansar cohort food frequency questionnaire (FFQ) to which a list of foods commonly used in Kermanshah was added. ${ }^{21}$ The food quantities were converted to grams using the manual for household measures and analyzed in the software Nutritionist 4 to obtain nutritional parameters (micronutrient, macronutrient, and energy intake). ${ }^{22}$ The cooking oils of animal origin used in the study area are butter and ghee. 
To assess the relationship of the use of butter and ghee with the risk of MetS and its components, the intake of these products was classified into 5 quintiles from very low (quintile 1) to very high (quintile 5). We calculated the ratio of reported energy intake to BMR (rEI: BMR) to evaluate the validity of energy intake. To match the relative degree of under- and over-reporting of energy, we briefly used the values defined by Goldberg et al which proposed by $\mathrm{FAO} / \mathrm{WHO} / \mathrm{UNU}$ for assessing the energy expenditure. According to these surveys, EI:RMR ratio less than 1.35 was not consistent with usual dietary intake and considered underreporting., ${ }^{23,24}$ In the current study we considered EI:BMR at ranges of 1.35-2.39 as normal reporting of energy intake. ${ }^{25}$

\section{Biochemical and Blood Pressure Assessment}

To evaluate the serum level of biochemical markers, blood samples were taken from the brachial vein after 12 hours of fasting. After centrifugation, serum samples were stored in the RaNCD cohort study laboratory at $-72^{\circ} \mathrm{C}$ until analysis. ${ }^{19}$ Serum total triglyceride (TG), total cholesterol, and High-density lipoprotein (HDL-C) levels were measured by routine laboratory methods ${ }^{26}$ (Pars Azmoon kit, Pars Azmoon Inc., Tehran, Iran). The Friedewald equation was used to calculate low-density lipoprotein (LDL-C) levels. ${ }^{27}$ Fasting blood glucose (FBG) was measured using glucose oxidase test. Systolic blood pressure (SBP) and diastolic blood pressure (DBP) were measured using a Reister sphygmomanometer after sitting for at least 4-5 minutes. This measurement was performed twice for each hand with 10-minute intervals and the average of the measurements was recorded as the person's final blood pressure. $^{19}$

\section{Anthropometric Assessment}

Assessed anthropometric parameters included height, weight, BMI, waist circumference, and hip circumference. Weight was measured with minimal clothing and without shoes using the InBody 770 device (Inbody Co., Seoul, Korea). Height was measured using the BSM 370 automatic measuring device (Biospace Co., Seoul, Korea) in the standing position without shoes with the shoulders held in their normal position. BMI was calculated. Waist and hip circumferences were also measured according to RaNCD protocols. ${ }^{19,20}$

\section{Definition of Metabolic Syndrome}

In the present study, MetS was defined as meeting at least three criteria specified in the Modified NCEP ATP III for Iranian adults. ${ }^{28}$ These criteria are as follows:

- $\mathrm{FBG} \geq 100 \mathrm{mg} / \mathrm{dl}$ or taking blood glucose-lowering medications

- Serum $\mathrm{TG} \geq 150 \mathrm{mg} / \mathrm{dl}$ or taking TG-lowering medications

- HDL-C $\leq 40 \mathrm{mg} / \mathrm{dl}$ in men and $\leq 50 \mathrm{mg} / \mathrm{dl}$ in women or taking HDL-C boosting medications

- $\mathrm{SBP} \geq 130 \mathrm{~mm} / \mathrm{Hg}$ or $\mathrm{DBP} \geq 85 \mathrm{~mm} / \mathrm{Hg}$ or taking blood pressure-lowering medications

- Waist circumference $\geq 95 \mathrm{~cm}$ for both genders.

\section{Definition of Kermanshah Ghee}

According to the definition of International Dairy Federation, ${ }^{29}$ ghee is a dairy product made from milk, cream or butter of cattle, sheep, or goat that contains $65 \%$ SFA and $33 \%$ MUFA. $^{30}$ Kermanshah ghee is a type of ghee produced by traditional methods in rural areas of Kermanshah province. To prepare this ghee, one first ferments the milk to produce yogurt and keeps it at room temperature for one night, then shakes the yogurt for a few hours in a classic round churning machine to separate the butterfat from the yogurt. After melting this butterfat and removing its impurities, the remained fat is called ghee that contains about $60.4 \%$ SFA, $31.4 \%$ MUFA, $4 \%$ PUFA, and $1.5 \%$ TFA. $^{31}$

\section{Data Analysis}

Data were analyzed using the software STATA 14 . The analysis of covariance (ANCOVA) was used to compare butter/ghee consumption quintiles (energy-, age-, and gender-adjusted) in terms of intake of nutrients and food groups. Upon encountering significant differences, the Tukey post hoc test was used to make pairwise comparisons between the groups. Logistic regression was used to check whether there is any correlation between the use of butter/ghee and the odds of having MetS. The correlation between the use of butter/ghee and the components of MetS was assessed by the use of linear regression. Regression models were used to eliminate the effect of confounding variables. In addition to the crude model, the analysis was performed with three other models with adjustment for significant variables: 
- Model 1: age, gender, education level, marital status, economic status, and physical activity

- Model 2: Additional adjustment for dietary intakes including fruits, vegetables, legumes, bread and cereals, dairy products, sugars, miscellaneous foods (spices and food supplements), oils and oilseeds, percentage of energy intake from fat except butter or ghee.

- Model 3: Additional adjustment for BMI>30 and hip circumference

In all multivariate models, the first quintile was considered as the reference. The relationship between the consumption of butter/ghee and the study variables was assessed using the crude and adjusted odds ratios taken from the logistic regression model at $95 \%$ confidence level. For all tests, the level of statistical significance was $\mathrm{P}$-value $<0.05$.

\section{Results}

Of the 5550 persons whose data met the inclusion/exclusion criteria, 53.8\% were female and the rest were male. The mean age of the subjects was $47.6 \pm 8.19$ years. The prevalence of MetS was $31.42 \%$. The mean intake of butter and Kermanshah ghee was $1.85 \pm 3.32$ and $2.32 \pm 5.13 \mathrm{~g} /$ day, respectively. The demographic characteristics of the subjects across quintiles of butter/ghee intakes after adjustment for total energy intake are given in Table 1. As this table shows, people in the highest quintile of butter intake had higher mean age, higher physical activity, and lower BMI than those in the lowest quintile. People in the highest quintile of ghee intake were mostly women and had lower BMI and obesity and more physical activity than the lowest quintile. Also, the use of butter and Kermanshah ghee had a significant correlation with socioeconomic status.

Table 2 shows the intake of nutrients and food groups in the butter/ghee consumption quintiles after adjustment for total energy intake, age, and gender. As can be seen, people in the highest quintile of butter consumption had higher cholesterol and intakes of energy, protein, fat, SFA, dairy, oils and oilseeds and lower intakes of carbohydrates, fiber, PUFA, vegetables, bread and cereals, and miscellaneous foods than the lowest quintile. Individuals in the highest quintiles of Kermanshah ghee consumption had higher cholesterol and intakes of energy, protein, fat, SFA, dairy, oils and oilseeds and lower intakes of carbohydrates, MUFA, PUFA, fiber, vegetables, legumes, bread and cereals and miscellaneous foods than the first quintile.
The odds ratio of the MetS and the geometric means of its components in the butter/ghee consumption quintiles are shown in Tables 3 and 4. In both crude and adjusted models, the geometric mean of TG in the highest quintile of butter consumption was significantly lower than that in the lowest quintile $(\mathrm{P}<0.05)$. A similar statistically significant relation was observed for the geometric mean of FBG in the crude model and Models 1 and 2. In Model 3 however (adjusted for BMI and hip circumference), this relation was not statistically significant $(\mathrm{P}=0.09)$. In all models, the geometric mean of HDL-C significantly increased with the quintile $(\mathrm{P}<0.05)$. There was no significant difference between the butter consumption quintiles in terms of the geometric mean of DBP or waist circumference $(\mathrm{P}>0.05)$. Only Model 1 showed a significant difference between the geometric means of SBP in the highest and lowest butter consumption quintiles $(\mathrm{P}<0.039)$ and even this difference became insignificant after further adjustment for confounding variables. In the crude model and Models 1 and 2, the odds of having MetS significantly decreased with the increase of butter consumption ( $\mathrm{P}=0.001)$. In Model 3, decrease in the odds of developing MetS was less pronounced but still statistically significant (26\% difference between the highest and lowest quintiles with $\mathrm{P}=0.007,95 \% \mathrm{CI}=0.59-0.92, \mathrm{OR}=0.74$ )

In the crude model and Models 1 and 2, the geometric mean of TG in the highest quintile of Kermanshah ghee consumption was significantly lower than that in the lowest quintile $(\mathrm{P}<0.05)$, but in Model 3 this difference although present was insignificant $(\mathrm{P}=0.405)$. In all models, the geometric mean of SBP and DBP decreased ( $\mathrm{P}$ $<0.001$ ) and the geometric mean of HDL-C increased ( $\mathrm{P}$ $<0.001$ ) with the increase of ghee consumption. None of the models showed a significant difference between the highest and lowest quintiles of ghee consumption in terms of the geometric mean of FBG $(\mathrm{P}>0.05)$. In all of the models, the geometric mean of waist circumference in the highest Kermanshah ghee quintiles was significantly lower than that in the lowest quintile $(\mathrm{P}<0.001)$. In the crude model and Models 1 and 2, the odds of having MetS significantly decreased with the increase of ghee consumption ( $\mathrm{P}<0.001)$. In Model 3 (adjustment of hip circumference and $\mathrm{BMI}$ ), this relation was weaker but still significant (22\% difference between the highest and lowest quintiles with $\mathrm{P}=0.045,95 \% \mathrm{CI}=0.61-0.99, \mathrm{OR}=0.78$ ).

\section{Discussion}

In this cross-sectional study on the adult population of Ravansar Non-Communicable Disease (RaNCD) cohort, 


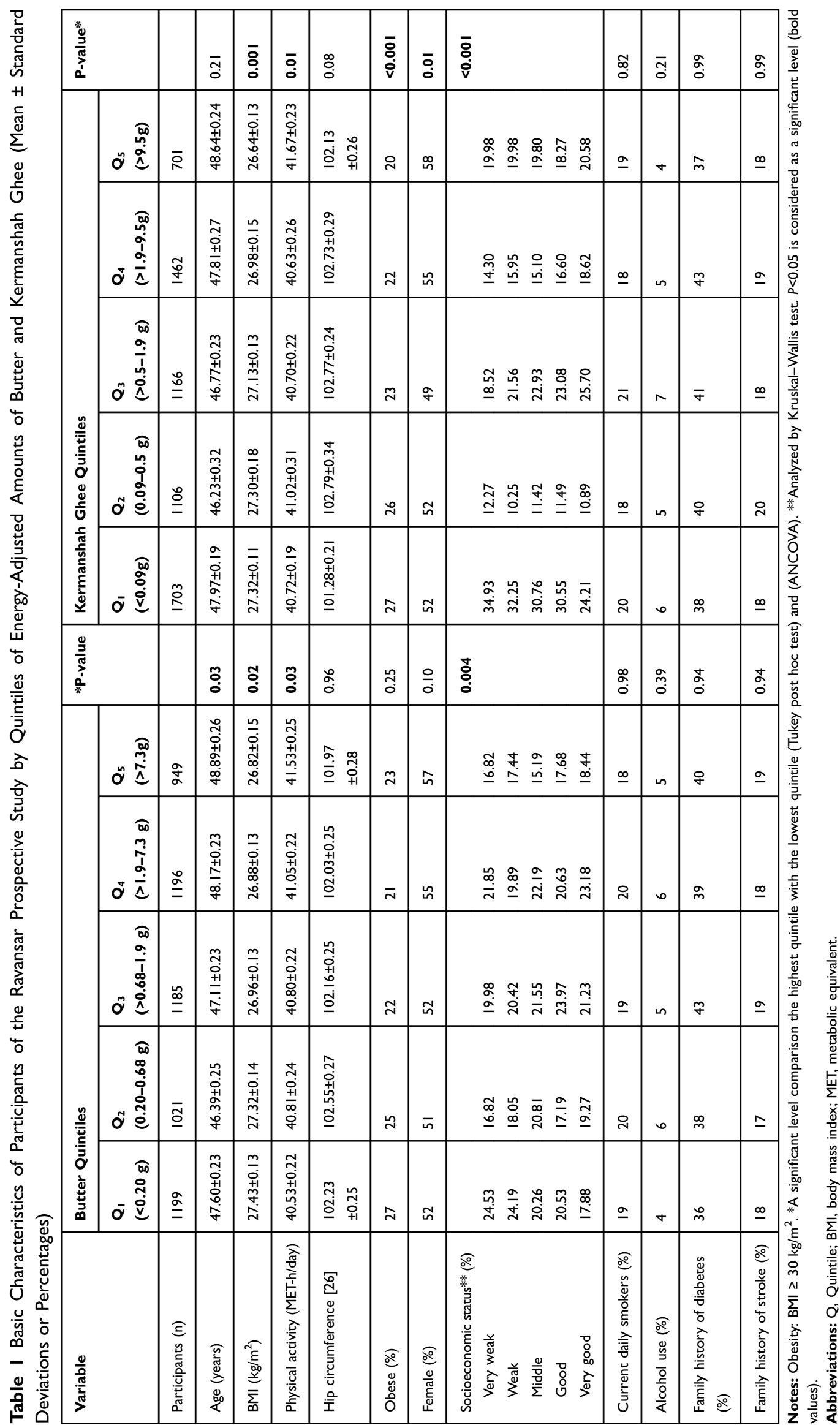




\begin{tabular}{|c|c|c|c|c|c|c|c|c|c|c|c|c|c|c|c|c|c|c|c|c|}
\hline 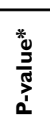 & & & $\begin{array}{l}\bar{o} \\
\dot{j} \\
\text { v }\end{array}$ & $\begin{array}{l}\bar{o} \\
\dot{v}\end{array}$ & iò & õ & $\begin{array}{l}\bar{o} \\
\dot{0}\end{array}$ & $\begin{array}{l}\bar{o} \\
\dot{d} \\
\mathrm{v}\end{array}$ & $\begin{array}{l}\bar{o} \\
\dot{0} \\
\mathrm{v}\end{array}$ & ڤั & $\begin{array}{l}\bar{o} \\
\dot{i}\end{array}$ & & $\hat{o}$ & $\begin{array}{l}\bar{o} \\
\dot{0}\end{array}$ & $\stackrel{\substack{\infty \\
\infty \\
\infty}}{0}$ & $\begin{array}{l}\bar{o} \\
\dot{0} \\
\mathrm{v}\end{array}$ & $\begin{array}{l}\bar{o} \\
\dot{0} \\
\mathrm{v}\end{array}$ & $\begin{array}{l}\bar{o} \\
\dot{i}\end{array}$ & $\begin{array}{l}\bar{o} \\
\dot{i} \\
\mathrm{v}\end{array}$ & 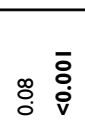 \\
\hline \multirow{5}{*}{ 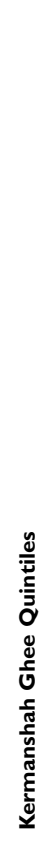 } & 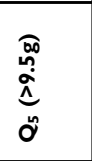 & $\bar{R}$ & 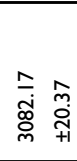 & 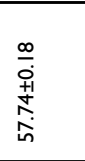 & 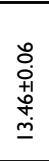 & 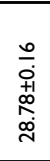 & 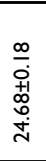 & 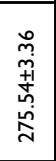 & $\begin{array}{l}\stackrel{0}{0} \\
\stackrel{1}{+1} \\
a\end{array}$ & $\begin{array}{l}\hat{0} \\
\text { +1 } \\
\text { d }\end{array}$ & 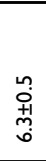 & & 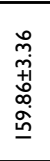 & 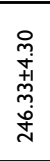 & 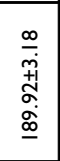 & 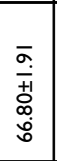 & 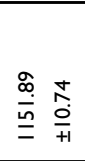 & 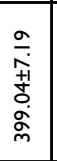 & 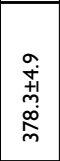 & 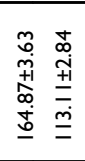 \\
\hline & ठ & 㞥 & 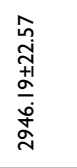 & 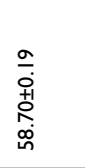 & 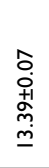 & $\begin{array}{l}\frac{\infty}{+1} \\
\text { 11 } \\
\stackrel{\alpha}{\alpha} \\
i\end{array}$ & 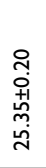 & 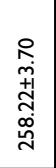 & $\begin{array}{l}\hat{0} \\
\text { îl } \\
\text { O1 } \\
\infty\end{array}$ & 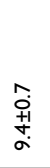 & 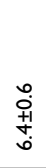 & & 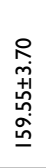 & 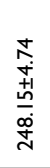 & 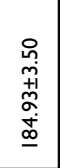 & 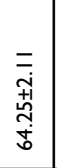 & 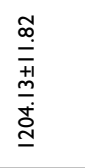 & 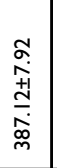 & 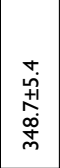 & 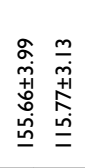 \\
\hline & 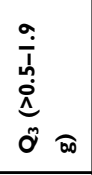 & $\stackrel{\circ}{=}$ & 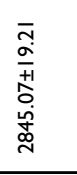 & 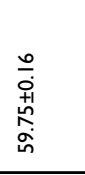 & 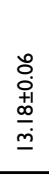 & $\begin{array}{l}\stackrel{n}{o n} \\
\text { ind } \\
\text { in } \\
\text { in }\end{array}$ & 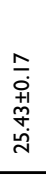 & 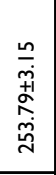 & $\begin{array}{l}\stackrel{0}{0} \\
\substack{1 \\
0 \\
\infty}\end{array}$ & $\begin{array}{l}\stackrel{\circ}{\circ} \\
\frac{+1}{a}\end{array}$ & $\begin{array}{l}\text { n. } \\
\text { 市 } \\
0 \\
0\end{array}$ & & 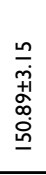 & 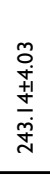 & 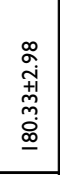 & 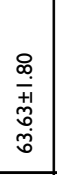 & $\begin{array}{l}\hat{0} \\
\stackrel{0}{+1} \\
+1 \\
\sigma \\
\infty \\
\stackrel{0}{a} \\
\underline{a}\end{array}$ & 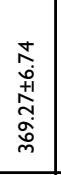 & $\begin{array}{l}0 \\
\stackrel{+}{+} \\
\stackrel{+}{+1} \\
0 \\
0 \\
\end{array}$ & 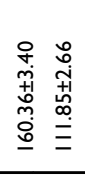 \\
\hline & 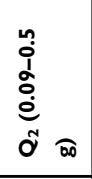 & $\stackrel{\circ}{\underline{\circ}}$ & 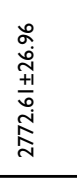 & $\begin{array}{l}\text { ָ̊ } \\
\text { 11 } \\
0 \\
0 \\
0\end{array}$ & 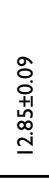 & 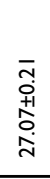 & 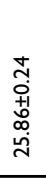 & 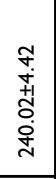 & $\begin{array}{l}\infty \\
\stackrel{+}{+} \\
\infty \\
\infty\end{array}$ & $\begin{array}{l}0 \\
\text { ò } \\
\text { İ } \\
\text { d }\end{array}$ & 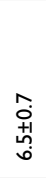 & & 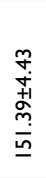 & $\begin{array}{l}\hat{\omega} \\
\dot{p} \\
+1 \\
m \\
m \\
\stackrel{n}{n}\end{array}$ & 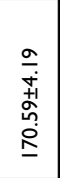 & 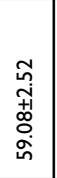 & 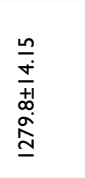 & 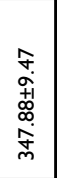 & $\begin{array}{l}n \\
0 \\
0 \\
01 \\
0 \\
0 \\
0 \\
m \\
m\end{array}$ & 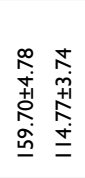 \\
\hline & $\begin{array}{l}\widehat{D} \\
\delta \\
\dot{0} \\
\dot{v} \\
\overline{0}\end{array}$ & $\stackrel{\varrho}{\underline{\Omega}}$ & 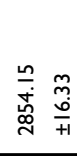 & 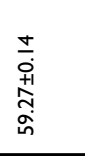 & 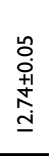 & 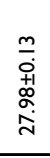 & 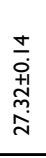 & 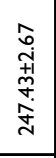 & 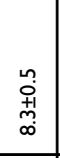 & $\begin{array}{l}n \\
0 \\
0+1 \\
0 \\
0\end{array}$ & $\begin{array}{l}+ \\
\text { 辛 } \\
\text { d. } \\
0\end{array}$ & & 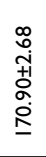 & 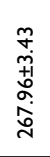 & 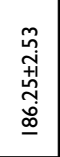 & 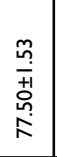 & 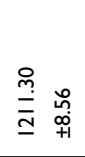 & 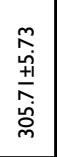 & 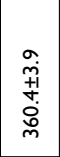 & 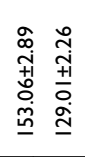 \\
\hline \multicolumn{2}{|l|}{ } & & $\begin{array}{l}\bar{\vdots} \\
\dot{i} \\
\dot{v}\end{array}$ & $\begin{array}{l}\bar{o} \\
\dot{0} \\
\dot{0}\end{array}$ & $\begin{array}{l}\bar{o} \\
\dot{0} \\
\mathrm{v}\end{array}$ & $\begin{array}{l}\bar{a} \\
\dot{j} \\
\dot{v}\end{array}$ & $\begin{array}{l}\bar{\vdots} \\
\dot{0} \\
\dot{v}\end{array}$ & $\begin{array}{l}\bar{\vdots} \\
\dot{j} \\
\dot{v}\end{array}$ & $\begin{array}{l}\bar{o} \\
\dot{0} \\
\mathrm{i}\end{array}$ & ঃ̊ & $\begin{array}{l}\bar{o} \\
\dot{0} \\
\dot{v}\end{array}$ & & $\overline{\bar{o}}$ & ö & oे & ల్ల్య & $\begin{array}{l}\bar{o} \\
\dot{0} \\
\text { v }\end{array}$ & $\begin{array}{l}\overline{\grave{o}} \\
\dot{\dot{v}}\end{array}$ & $\begin{array}{l}\overline{\grave{o}} \\
\dot{\dot{v}}\end{array}$ & $\frac{\infty}{0} \stackrel{0}{0}$ \\
\hline \multirow{5}{*}{ 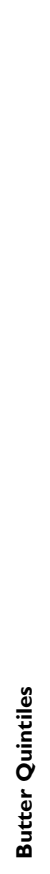 } & $\begin{array}{l}\stackrel{0}{0} \\
\stackrel{0}{\hat{n}} \\
\ddot{0}\end{array}$ & gे & 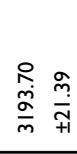 & 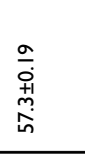 & 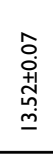 & 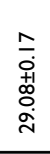 & 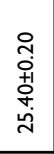 & 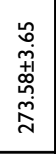 & $\begin{array}{l}\hat{j} \\
\text { 京 } \\
\alpha\end{array}$ & 突 & 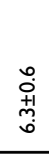 & & 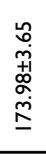 & 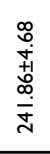 & 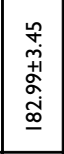 & 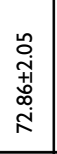 & 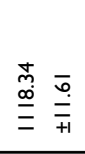 & 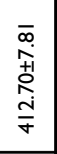 & 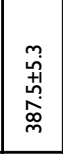 & 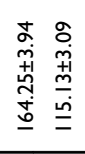 \\
\hline & 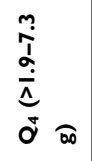 & $\stackrel{\circ}{=}$ & 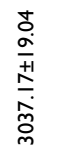 & 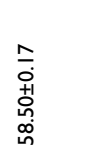 & 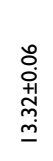 & 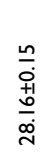 & 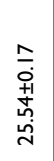 & 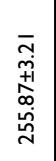 & $\begin{array}{l}\stackrel{0}{0} \\
\text { ị } \\
\text { a. }\end{array}$ & 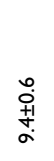 & 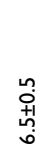 & & 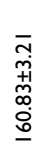 & 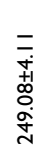 & 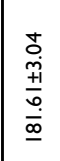 & 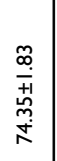 & $\begin{array}{l}\bar{y} \\
\stackrel{\bar{\delta}}{+1} \\
+1 \\
\underline{0} \\
\stackrel{\infty}{=} \\
\underline{=}\end{array}$ & 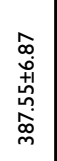 & 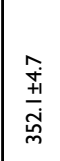 & 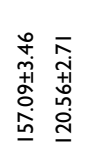 \\
\hline & 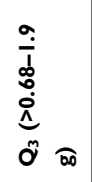 & $\stackrel{\stackrel{\omega}{0}}{=}$ & 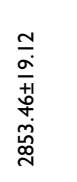 & 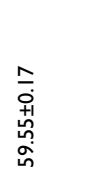 & 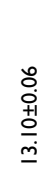 & 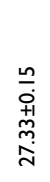 & 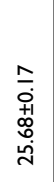 & 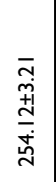 & 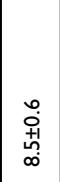 & $\begin{array}{l}\stackrel{\circ}{+1} \\
\text { †ू }\end{array}$ & 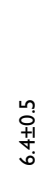 & & 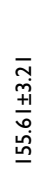 & 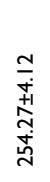 & 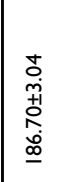 & 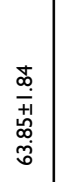 & 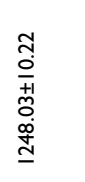 & 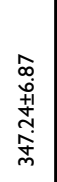 & $\begin{array}{l}\stackrel{+}{+} \\
\stackrel{+1}{\infty} \\
\stackrel{m}{m}\end{array}$ & 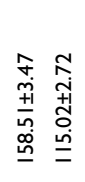 \\
\hline & 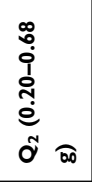 & $\overline{\underline{\underline{\sigma}}}$ & 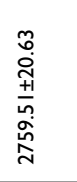 & 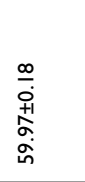 & 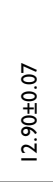 & 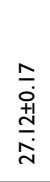 & 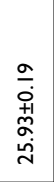 & 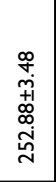 & $\begin{array}{l}\stackrel{0}{0} \\
\stackrel{1}{1} \\
\infty\end{array}$ & $\begin{array}{l}\hat{0} \\
\stackrel{+}{+} \\
\text { d }\end{array}$ & 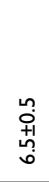 & & 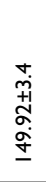 & 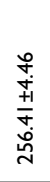 & 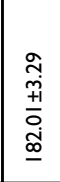 & $\begin{array}{l}\frac{\infty}{0} \\
\frac{0}{+1} \\
\frac{+\infty}{\infty} \\
\frac{0}{0}\end{array}$ & 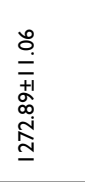 & 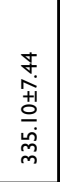 & 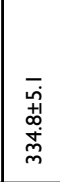 & 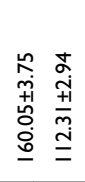 \\
\hline & 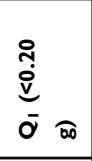 & $\stackrel{\sigma}{=}$ & 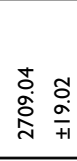 & 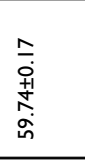 & $\begin{array}{l}\stackrel{0}{0} \\
\text { ì } \\
\stackrel{1}{0} \\
\text { I. }\end{array}$ & 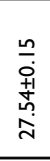 & 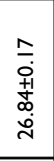 & 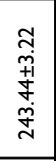 & \begin{tabular}{|l|}
0 \\
$\dot{0}$ \\
$\frac{1}{\infty}$ \\
$\infty$
\end{tabular} & 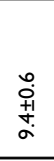 & $\begin{array}{l}n \\
0 \\
01 \\
0 \\
0\end{array}$ & & 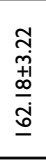 & 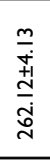 & 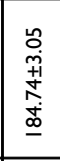 & 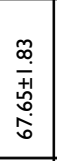 & 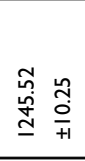 & 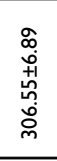 & 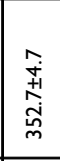 & 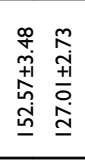 \\
\hline & 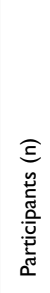 & 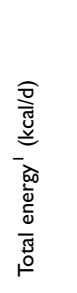 & 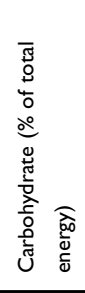 & 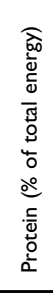 & 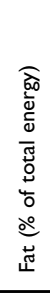 & 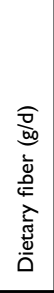 & 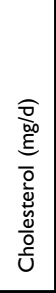 & 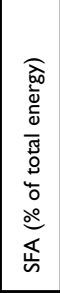 & 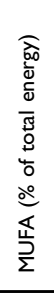 & 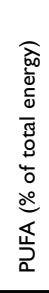 & 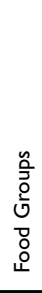 & 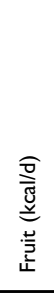 & 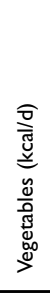 & 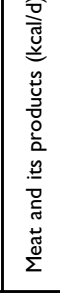 & 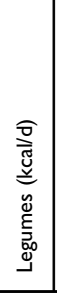 & 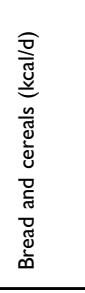 & 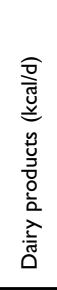 & 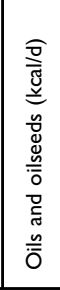 & 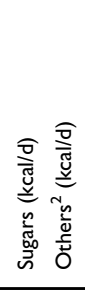 \\
\hline
\end{tabular}


Table 3 Multivariate Adjusted Odds Ratio $(95 \% \mathrm{Cl})$ for Metabolic Syndrome and Geometric Means of Its Components Across Quintile Categories of Butter Among Participants of the Ravansar Prospective Study

\begin{tabular}{|c|c|c|c|c|c|c|}
\hline \multirow[t]{2}{*}{ Variable } & \multicolumn{5}{|c|}{ Butter Quintiles } & \multirow[t]{2}{*}{ *P- value } \\
\hline & $Q_{1}(n=1199)$ & $Q_{2}(n=\mid 021)$ & $Q_{3}(n=1 \mid 85)$ & $Q_{4}(n=1196)$ & $Q_{5}(n=949)$ & \\
\hline \multicolumn{7}{|l|}{$\mathrm{TG}^{\prime}(\mathrm{mg} / \mathrm{dl})$} \\
\hline Crude & $|40.1| \pm 2.28$ & $|30.8| \pm 2.47$ & $134.24 \pm 2.29$ & $136.02 \pm 2.28$ & $126.72 \pm 2.56$ & $<0.001$ \\
\hline Model I & $140.13 \pm 2.25$ & $131.05 \pm 2.44$ & $134.27 \pm 2.26$ & $135.76 \pm 2.25$ & $126.73 \pm 2.53$ & $<0.001$ \\
\hline Model 2 & $139.66 \pm 2.30$ & $130.83 \pm 2.46$ & $|34.0| \pm 2.26$ & $135.69 \pm 2.27$ & $127.98 \pm 2.66$ & 0.003 \\
\hline Model 3 & $137.54 \pm 2.23$ & $129.50 \pm 2.38$ & $134.57 \pm 2.19$ & $137.01 \pm 2.19$ & $129.74 \pm 2.57$ & 0.026 \\
\hline \multicolumn{7}{|l|}{$\mathrm{FBG}^{\prime}(\mathrm{mg} / \mathrm{dl})$} \\
\hline Crude & $93.01 \pm 0.47$ & $91.54 \pm 0.51$ & $91.84 \pm 0.47$ & $92.56 \pm 0.47$ & $91.56 \pm 0.53$ & 0.042 \\
\hline Model I & $92.96 \pm 0.47$ & $91.76 \pm 0.51$ & $91.94 \pm 0.47$ & $92.44 \pm 0.47$ & $91.41 \pm 0.53$ & 0.030 \\
\hline Model 2 & $93.11 \pm 0.48$ & $91.71 \pm 0.51$ & $91.88 \pm 0.47$ & $92.36 \pm 0.47$ & $91.46 \pm 0.55$ & 0.006 \\
\hline Model 3 & $92.89 \pm 0.47$ & $91.55 \pm 0.50$ & $91.95 \pm 0.46$ & $92.51 \pm 0.46$ & $91.64 \pm 0.54$ & 0.018 \\
\hline \multicolumn{7}{|l|}{$\mathrm{DBP}^{\prime}(\mathrm{mmHg})$} \\
\hline Crude & $69.98 \pm 0.28$ & $68.90 \pm 0.30$ & $69.26 \pm 0.28$ & $69.34 \pm 0.28$ & $69.64 \pm 0.31$ & 0.435 \\
\hline Model I & $69.89 \pm 0.27$ & $69.15 \pm 0.29$ & $69.39 \pm 0.27$ & $69.24 \pm 0.27$ & $69.45 \pm 0.30$ & 0.291 \\
\hline Model 2 & $69.78 \pm 0.28$ & $69.18 \pm 0.29$ & $69.4 I \pm 0.27$ & $69.23 \pm 0.27$ & $69.54 \pm 0.32$ & 0.961 \\
\hline Model 3 & $69.54 \pm 0.27$ & $69.03 \pm 0.29$ & $69.48 \pm 0.26$ & $69.38 \pm 0.26$ & $69.74 \pm 0.31$ & 0.346 \\
\hline \multicolumn{7}{|l|}{$\mathrm{SBP}^{\prime}(\mathrm{mmHg})$} \\
\hline Crude & $108.58 \pm 0.48$ & $106.70 \pm 0.52$ & $107.11 \pm 0.49$ & $107.17 \pm 0.48$ & $107.46 \pm 0.54$ & 0.128 \\
\hline Model I & $|08.4| \pm 0.46$ & $107.33 \pm 0.49$ & $107.39 \pm 0.46$ & $106.90 \pm 0.45$ & $|06.98 \pm 0.5|$ & 0.039 \\
\hline Model 2 & $108.18 \pm 0.47$ & $107.32 \pm 0.50$ & $107.39 \pm 0.46$ & $106.91 \pm 0.46$ & $107.27 \pm 0.54$ & 0.398 \\
\hline Model 3 & $107.80 \pm 0.45$ & $107.07 \pm 0.48$ & $107.50 \pm 0.44$ & $107.15 \pm 0.44$ & $107.58 \pm 0.52$ & 0.921 \\
\hline \multicolumn{7}{|l|}{$\mathrm{HDL}-\mathrm{C}^{\prime}(\mathrm{mg} / \mathrm{dl})$} \\
\hline Crude & $46.39 \pm 0.33$ & $46.92 \pm 0.35$ & $46.76 \pm 0.33$ & $47.38 \pm 0.33$ & $47.94 \pm 0.37$ & 0.002 \\
\hline Model I & $46.09 \pm 0.31$ & $47.03 \pm 0.33$ & $46.85 \pm 0.31$ & $47.47 \pm 0.31$ & $47.98 \pm 0.35$ & $<0.001$ \\
\hline Model 2 & $46.14 \pm 0.31$ & $46.90 \pm 0.34$ & $46.74 \pm 0.31$ & $47.52 \pm 0.31$ & $48.13 \pm 0.36$ & 0.002 \\
\hline Model 3 & $46.45 \pm 0.30$ & $47.08 \pm 0.33$ & $46.66 \pm 0.30$ & $47.33 \pm 0.30$ & $47.88 \pm 0.35$ & 0.034 \\
\hline \multicolumn{7}{|l|}{$W C^{\prime}(\mathrm{cm})$} \\
\hline Crude & $96.74 \pm 0.30$ & $96.74 \pm 0.33$ & $96.36 \pm 0.30$ & $96.62 \pm 0.30$ & $96.56 \pm 0.34$ & 0.690 \\
\hline Model I & $96.65 \pm 0.30$ & $96.72 \pm 0.32$ & $96.36 \pm 0.30$ & $96.63 \pm 0.30$ & $96.67 \pm 0.33$ & 0.964 \\
\hline Model 2 & $96.96 \pm 0.30$ & $96.85 \pm 0.32$ & $96.34 \pm 0.30$ & $96.47 \pm 0.30$ & $96.38 \pm 0.35$ & 0.300 \\
\hline Model 3 & $96.58 \pm 0.14$ & $96.47 \pm 0.15$ & $96.5 I \pm 0.13$ & $96.76 \pm 0.14$ & $96.69 \pm 0.16$ & 0.905 \\
\hline \multicolumn{7}{|l|}{$* * M e t S^{2}$} \\
\hline Crude & I & $0.74(0.62-0.89)$ & $0.77(0.65-0.91)$ & $0.80(0.68-0.95)$ & $0.72(0.60-0.87)$ & 0.001 \\
\hline Model I & I & $0.76(0.63-0.91)$ & $0.78(0.65-0.93)$ & $0.80(0.67-0.95)$ & $0.72(0.60-0.87)$ & 0.001 \\
\hline Model 2 & I & $0.76(0.63-0.91)$ & $0.78(0.65-0.93)$ & $0.78(0.65-0.93)$ & $0.7 \mathrm{I}(0.58-0.87)$ & 0.001 \\
\hline Model 3 & I & $0.74(0.60-0.90)$ & $0.82(0.67-0.99)$ & $0.84(0.69-1.03)$ & $0.74(0.59-0.92)$ & 0.007 \\
\hline
\end{tabular}

Notes: 'Geometric mean \pm standard error. ${ }^{2}$ Odds ratio $(95 \%$ confidence interval). $* A$ significant level comparison the highest quintile with the lowest quintile of butter (logistic and linear regression). ${ }^{* *} \mathrm{~A}$ significant level of additive or decreasing trend in quintiles of butter ( $\mathrm{p}$-trends for MetS were significant in all models except model3). $p<0.05$ was considered as a significant level (bold values). Modell was adjusted for age (in y), physical activity (continuous), socioeconomic status (categorical), current use of medications (except use of hypertriglyceridemic, hypertensive and hyperglycemic medications), Model 2 was adjusted for variables in Model $\mathrm{I}$ and for dietary intakes including vegetables, bread and cereals, dairy products, others (spices and food supplements), oils and oilseeds, percentage of energy from fat except butter. Model3 was adjusted for variables in Model 2 and for BMI and Hip circumference which may be mediators as well as confounders (continuous).

Abbreviations: Q, quintile; TG, triglyceride; FBG, fasting blood glucose; DBP, diastolic blood pressure; SBP, systolic blood pressure; HDL-C, high-density lipoprotein; WC, waist circumference; MetS, metabolic syndrome.

we observed a strong and inverse relationship with a linear dose-response pattern between the consumption of Kermanshah ghee and butter (ghee/butter) with the odds of having MetS after adjusting of cofounders. Also, some components of MetS improved significantly with the greater consumption of ghee/butter. 
Table 4 Multivariate Adjusted Odds Ratio $(95 \% \mathrm{Cl})$ for Metabolic Syndrome and Geometric Means of Its Components Across Quintile Categories of Kermanshah Ghee Among Participants of the Ravansar Prospective Study

\begin{tabular}{|c|c|c|c|c|c|c|}
\hline \multirow[t]{2}{*}{ Variable } & \multicolumn{5}{|c|}{ Kermanshah Ghee Quintiles } & \multirow[t]{2}{*}{ *P- value } \\
\hline & $Q_{1}(n=1703)$ & $Q_{2}(n=1106)$ & $Q_{3}(n=1166)$ & $Q_{4}(n=\mid 462)$ & $Q_{5}(n=70 I)$ & \\
\hline $\begin{array}{l}\text { TG' }(\mathrm{mg} / \mathrm{dl}) \\
\text { Crude } \\
\text { Model I } \\
\text { Model } 2 \\
\text { Model } 3\end{array}$ & $\begin{array}{l}|4| .27 \pm|.9| \\
|40.76 \pm| .89 \\
\mid 40.43 \pm 1.93 \\
\mid 37.78 \pm 1.89\end{array}$ & $\begin{array}{l}129.52 \pm 3.15 \\
130.50 \pm 3.11 \\
130.04 \pm 3.13 \\
129.28 \pm 3.03\end{array}$ & $\begin{array}{l}134.27 \pm 2.24 \\
133.59 \pm 2.22 \\
133.49 \pm 2.23 \\
133.33 \pm 2.16\end{array}$ & $\begin{array}{l}129.04 \pm 2.64 \\
\mid 29.07 \pm 2.61 \\
128.96 \pm 2.61 \\
\mid 30.91 \pm 2.54\end{array}$ & $\begin{array}{l}128.86 \pm 2.38 \\
\mid 29.85 \pm 2.36 \\
\mid 30.82 \pm 2.44 \\
\mid 33.96 \pm 2.37\end{array}$ & $\begin{array}{l}<0.001 \\
<0.001 \\
0.032 \\
0.405\end{array}$ \\
\hline $\begin{array}{c}\text { FBG }^{\prime}(\mathrm{mg} / \mathrm{dl}) \\
\text { Crude } \\
\text { Model I } \\
\text { Model } 2 \\
\text { Model } 3\end{array}$ & $\begin{array}{l}91.47 \pm 0.39 \\
91.36 \pm 0.39 \\
91.62 \pm 0.40 \\
91.48 \pm 0.40\end{array}$ & $\begin{array}{l}93.28 \pm 0.65 \\
93.64 \pm 0.65 \\
93.55 \pm 0.65 \\
93.40 \pm 0.64\end{array}$ & $\begin{array}{l}92.37 \pm 0.46 \\
92.49 \pm 0.46 \\
92.38 \pm 0.46 \\
92.29 \pm 0.45\end{array}$ & $\begin{array}{l}92.97 \pm 0.55 \\
92.91 \pm 0.54 \\
92.76 \pm 0.54 \\
92.90 \pm 0.53\end{array}$ & $\begin{array}{l}91.62 \pm 0.49 \\
91.52 \pm 0.49 \\
91.40 \pm 0.50 \\
91.70 \pm 0.50\end{array}$ & $\begin{array}{l}0.815 \\
0.805 \\
0.770 \\
0.444\end{array}$ \\
\hline $\begin{array}{l}\mathrm{DBP}^{\prime}(\mathrm{mmHg}) \\
\text { Crude } \\
\text { Model I } \\
\text { Model } 2 \\
\text { Model } 3\end{array}$ & $\begin{array}{l}70.73 \pm 0.23 \\
70.56 \pm 0.22 \\
70.53 \pm 0.23 \\
70.22 \pm 0.23\end{array}$ & $\begin{array}{l}69.13 \pm 0.39 \\
69.45 \pm 0.37 \\
69.52 \pm 0.38 \\
69.44 \pm 0.37\end{array}$ & $\begin{array}{l}68.79 \pm 0.27 \\
69.00 \pm 0.26 \\
69.09 \pm 0.27 \\
69.08 \pm 0.26\end{array}$ & $\begin{array}{l}69.35 \pm 0.32 \\
69.35 \pm 0.31 \\
69.33 \pm 0.31 \\
69.56 \pm 0.31\end{array}$ & $\begin{array}{l}68.37 \pm 0.29 \\
68.22 \pm 0.28 \\
68.14 \pm 0.29 \\
68.50 \pm 0.28\end{array}$ & $\begin{array}{l}<0.001 \\
<0.001 \\
<0.001 \\
<0.001\end{array}$ \\
\hline $\begin{array}{l}\mathrm{SBP}^{\prime}(\mathrm{mmHg}) \\
\text { Crude } \\
\text { Model I } \\
\text { Model } 2 \\
\text { Model } 3\end{array}$ & $\begin{array}{l}109.44 \pm 0.40 \\
109.10 \pm 0.38 \\
109.03 \pm 0.39 \\
108.66 \pm 0.38\end{array}$ & $\begin{array}{l}107.05 \pm 0.67 \\
107.85 \pm 0.63 \\
107.92 \pm 0.63 \\
107.75 \pm 0.61\end{array}$ & $\begin{array}{l}106.80 \pm 0.47 \\
107.23 \pm 0.45 \\
107.33 \pm 0.45 \\
107.26 \pm 0.44\end{array}$ & $\begin{array}{l}107.18 \pm 0.56 \\
107.11 \pm 0.53 \\
107.09 \pm 0.53 \\
107.39 \pm 0.51\end{array}$ & $\begin{array}{l}105.40 \pm 0.50 \\
105.04 \pm 0.48 \\
105.01 \pm 0.49 \\
105.54 \pm 0.48\end{array}$ & $\begin{array}{l}<0.001 \\
<0.001 \\
<0.001 \\
<0.001\end{array}$ \\
\hline $\begin{array}{l}\text { HDL-C' }(\mathrm{mg} / \mathrm{dl}) \\
\text { Crude } \\
\text { Model I } \\
\text { Model } 2 \\
\text { Model } 3\end{array}$ & $\begin{array}{l}45.34 \pm 0.27 \\
45.28 \pm 0.26 \\
45.42 \pm 0.26 \\
45.90 \pm 0.26\end{array}$ & $\begin{array}{l}47.40 \pm 0.45 \\
47.35 \pm 0.42 \\
47.22 \pm 0.43 \\
47.29 \pm 0.41\end{array}$ & $\begin{array}{l}46.86 \pm 0.32 \\
47.26 \pm 0.30 \\
47.11 \pm 0.30 \\
47.08 \pm 0.29\end{array}$ & $\begin{array}{l}48.27 \pm 0.38 \\
48.21 \pm 0.35 \\
48.17 \pm 0.36 \\
47.84 \pm 0.35\end{array}$ & $\begin{array}{l}48.72 \pm 0.34 \\
48.43 \pm 0.32 \\
48.49 \pm 0.33 \\
48.01 \pm 0.32\end{array}$ & $\begin{array}{l}<0.001 \\
<0.001 \\
<0.001 \\
0.013\end{array}$ \\
\hline $\begin{array}{l}\text { WC }(\mathrm{cm}) \\
\text { Crude } \\
\text { Model I } \\
\text { Model } 2 \\
\text { Model } 3\end{array}$ & $\begin{array}{l}95.70 \pm 0.25 \\
95.62 \pm 0.25 \\
95.82 \pm 0.25 \\
96.21 \pm 0.12\end{array}$ & $\begin{array}{l}96.81 \pm 0.42 \\
96.95 \pm 0.41 \\
96.97 \pm 0.41 \\
96.43 \pm 0.19\end{array}$ & $\begin{array}{l}97.11 \pm 0.30 \\
97.20 \pm 0.29 \\
97.18 \pm 0.29 \\
96.67 \pm 0.13\end{array}$ & $\begin{array}{l}97.53 \pm 0.35 \\
97.35 \pm 0.34 \\
97.21 \pm 0.34 \\
97.16 \pm 0.16\end{array}$ & $\begin{array}{l}96.55 \pm 0.32 \\
96.64 \pm 0.31 \\
96.46 \pm 0.32 \\
96.78 \pm 0.15\end{array}$ & $\begin{array}{l}0.040 \\
0.012 \\
0.089 \\
0.007\end{array}$ \\
\hline $\begin{array}{l}* * \text { MetS }{ }^{2} \\
\text { Crude } \\
\text { Model I } \\
\text { Model } 2 \\
\text { Model } 3\end{array}$ & $\begin{array}{l}\text { I } \\
\text { I } \\
\text { I } \\
\text { I }\end{array}$ & $\begin{array}{l}0.78(0.64-0.95) \\
0.81(0.66-0.99) \\
0.82(0.67-1.01) \\
0.81(0.66-0.99)\end{array}$ & $\begin{array}{l}0.89(0.76-1.04) \\
0.90(0.77-1.06) \\
0.91(0.78-1.07) \\
0.90(0.77-1.06)\end{array}$ & $\begin{array}{l}0.78(0.66-0.93) \\
0.77(0.64-0.93) \\
0.78(0.65-0.93) \\
0.77(0.64-0.93)\end{array}$ & $\begin{array}{l}0.7 I(0.60-0.84) \\
0.78(0.6 \mathrm{I}-0.99) \\
0.72(0.6 \mathrm{I}-0.85) \\
0.78(0.6 \mathrm{I}-0.99)\end{array}$ & $\begin{array}{l}<0.001 \\
<0.001 \\
<0.001 \\
0.045\end{array}$ \\
\hline
\end{tabular}

Notes: 'Geometric mean \pm standard error. ${ }^{2}$ Odds ratio ( $95 \%$ confidence interval). $* A$ significant level comparison the highest quintile with the lowest quintile of Kermanshah ghee (logistic and linear regression). **A significant level of additive or decreasing trend in quintiles of Kermanshah ghee ( $\mathrm{p}$-trends for MetS were significant in all models). $p<0.05$ was considered as a significant level (bold values). Modell was adjusted for gender (male/female), physical activity (continuous), socioeconomic status (categorical), current use of medications (except use of hypertriglyceridemic, hypertensive and hyperglycemic medications), Model2 was adjusted for variables in Model I and for dietary intakes including fruits, vegetables, legumes, bread and cereals, dairy products, sugars, others (spices and food supplements), oils and oilseeds, percentage of energy from fat except ghee. Model3 was adjusted for variables in Model 2 and for BMI and Hip circumference which may be mediators as well as confounders (continuous).

Abbreviations: Q, quintile; TG, triglyceride; FBG, fasting blood glucose; DBP, diastolic blood pressure; SBP, systolic blood pressure; HDL-C, high-density lipoprotein; WC, waist circumference; MetS, metabolic syndrome.

The present study showed that with increasing the Kermanshah ghee intake, DBP, SBP and waist circumference decreased and HDL-C increased significantly. Mahmoudifard et al reported that a ghee-containing diet significantly reduced TG levels and increased HDL. ${ }^{29}$ Other authors indicated that ghee as compared to sunflower oil decreases the weight and 
oxidative stress and had protective effects on the liver in rats. ${ }^{32}$ Also, long-term (5 months) consumption of yogurt butter oil as compared to flaxseed oil had no adverse effect on lipid profile and liver function in rats. ${ }^{33}$ The Possible mechanism of HDL elevation with consumption of Kermanshah ghee could be related to high levels of MUFA, especially oleic acid in this product. ${ }^{34}$ Researches have shown that MUFA-rich diets are associated with lower blood pressure. ${ }^{35}$ The decrease in plasma TG can be attributed to the antiatherogenic effect of conjugated linoleic acid (CLA) in milk fat. Loss of weight and subsequent reduction in waist circumference can be due to the high amounts of SFA and short- and medium-chain TG that are directly transported through the bloodstream to the liver and prevent obesity by increasing PPAR $\gamma$ gene expression. Also, CLA in milk fat can prevent from obesity by reducing the accumulation of visceral and ectopic fat. ${ }^{36}$

Hosseini et al found out rabbits receiving an animal oil diet had higher total cholesterol and LDL levels than those fed with hydrogenated and olive oils. ${ }^{15}$ Also, another study showed that replacement of $10 \%$ of the diet with animal oil for 4 weeks had no effect on total cholesterol level and hepatic lipid peroxidation but increased serum TG. ${ }^{16}$ Najafi et al reported that consumption of 30 grams of Kermanshah ghee per day instead of other oils for 1 month had no significant effect on serum lipid profile of healthy men. ${ }^{37}$ The discrepancy between our results and some of the previous reports may be attributed to the differences in the processing methods of ghee production. Kermanshah ghee is traditionally produced from yogurt (fermented dairy products) and has different fatty acids pattern than butter and oils that are directly extracted from milk, its production process results in higher amounts of short- and medium-chain fatty acids and lower amounts of cholesterol and long-chain fatty acids. ${ }^{31}$

In the current study with increasing butter intake the TG and FBG levels decreased significantly. Also, a positive relationship with a monotonic trend was observed between butter intake and elevation of HDL-C levels. The odds of having MetS significantly decreased with more consumption of butter. A prospective study indicated that high-fat dairy consumption was inversely associated with DBP, SBP, total cholesterol, and increased HDL level. ${ }^{17}$ Also, in a cross-over study, the consumption of dairy products for 6 weeks improved liver function and systemic inflammation among individuals with MetS. ${ }^{38}$ Another study also indicated that high intake of dairy fat decreases the risk of T2D and cardiovascular diseases. ${ }^{39}$ A meta-analysis has also reported that butter consumption significantly decreases the average incidence of T2D but is not associated with CVD events. ${ }^{40}$ In another metaanalysis of clinical trials, high-fat dairy consumption was inversely associated with T2D and the low-fat dairy product intake was related to the risk of the disease. ${ }^{41}$ In a study by Gagliardi et al on people with MetS, butter consumption for 5 weeks did not show any significant effect on HDL-C, TG, SBP, DBP, and inflammatory markers. $^{42}$ The study of Ari Palomäki et al on people with MetS showed that using a butter-containing diet lasting for 6 to 8 weeks made no significant difference in HDL-C, TG, and FBG levels, however, increased DBP and SBP although the changes were not statistically significant. $^{43}$

Regarding the possible causes and mechanisms responsible for our findings as well as the similar investigations, it can be argued that in addition to the content of butter that $50 \%$ of its dry weight is made of SFAs ${ }^{44}$ SFAs with an odd number of carbon atoms, which are mostly found in dairy fats such as butter, increase the fluidity of the vascular cell membrane and are associated with a reduced risk of T2D and MetS. ${ }^{39}$ Butter also contains short- and medium-chain SFAs such as lauric acid and myristic acid, which are known to have protective effects against T2D. ${ }^{44}$ Trans-palmitoleic acid, which is a biomarker of dairy fat and butter consumption, is inversely associated with T2D. ${ }^{35}$ Also, a research has shown that the butyrate of butter induces insulin sensitivity ${ }^{45}$ and its CLA has beneficial effects on metabolic diseases. ${ }^{36}$ Conversely, Lichtenstein et al found that consumption of butter was associated with increased FBG and HDL-C, although it had no effect on SBP and $\mathrm{DBP}^{34}$ Compared to present study, Lichtenstein et al study was performed on older population (mean age: 67 years) with a large percentage of postmenopausal women; it can be claimed that hormonal changes are associated with increased insulin resistance. Judd et al found that butter intake in adults with normal blood lipid levels has no effect on HDL-C and TG but can increase total cholesterol. ${ }^{44}$ As we know SFA consumption can increase both HDL-C and LDL-C. In Judd's study, more than $20 \%$ of consumed calorie was from SFA, but in our study even in the highest quintiles, SFA accounted for less than $10 \%$ of daily intake of calories. In a 28-day interventional study on postmenopausal women, the butter group with $20 \%$ SFA content in their diet had significantly higher total cholesterol and LDL-C than the olive oil group consuming $10 \%$ SFA; there was no difference between two groups in terms of DBP and SBP, 
FBG and $\mathrm{TG}^{46}$ The probable reasons for the difference between some of these findings and our results are that women in the mentioned study had android obesity (BMI $=30.4 \mathrm{~kg} / \mathrm{m}^{2}$ ), older age (mean age $=59.5$ years), and higher waist circumference (mean $102.1 \mathrm{~cm}$ ) than our subjects. It is well known that hormonal and sedentary impacts of menopause are associated with glucose intolerance, T2D, dyslipidemia, hypertension, and increased risk of cardiovascular diseases. ${ }^{46}$ In one prospective study, during 3 years of follow-up, investigators observed that butter consumption led to increase TG and decrease HDL$\mathrm{C}$ levels and was directly associated with the risk of MetS. ${ }^{7}$ Although this report contradicts our findings, it should be noted that the medium butter consumption in that study was $23.5 \mathrm{~g} / \mathrm{day}$, which is many times higher than the $1.8 \mathrm{~g}$ /day butter intake in ours.

One of the strengths of this study compared to previous works which are mostly focused on fat subclasses (SFA, MUFA, PUFA, and TFA) is attention to the pattern of consumption; it makes the results more suitable for presentation to the public as practical health advice.

The present study also had several limitations that should be mentioned. Firstly, given the questionnairebased nature of this study, the results may have been affected by recall bias and information bias. Also, like other observational studies, it is impossible to mention a cause-and-effect relationship. Although much effort was made to minimize the effect of confounding variables, residual confounders, whose effects cannot be ignored, may still influence the results.

\section{Conclusions}

This study indicated the inverse relationship between Kermanshah ghee and butter consumption with MetS and some of its components. Therefore, consumption of milkbased oils may be associated with lower cardiovascular risk factors. However, clinical trials are required to recommend the most effective dosages.

\section{Abbreviations}

APO A-1, apolipoprotein A-1; BMI, body mass index; CHD, coronary heart disease; CLA, conjugated linoleic acid; CVD, cardiovascular disease; DBP, diastolic blood pressure; FBG, fasting blood glucose; FFQ, Food Frequency Questionnaire; HDL, high density lipoprotein; IDF, International Dairy Federation; LDL, low density lipoprotein; MET, metabolic equivalent of task; MUFA, mono unsaturated fatty acid; PUFA, poly unsaturated fatty acid; RaNCD, Ravansar Non-
Communicable Diseases; SBP, systolic blood pressure; SFA, saturated fatty acid; T2D, Type 2 diabetes; TFA, trans-fatty acids; TG, triglyceride.

\section{Data Sharing Statement}

The datasets (RaNCD) used and/or analyzed during the current study are available in the manuscript and from the corresponding author on reasonable request.

\section{Ethics and Consent Statement}

This study was approved by the Ethical Board (IR.KUMS. REC.1396.532) and supported by a research grant of Kermanshah University of Medical Sciences Research Council. The ethical consideration involved Helsinki declaration statement of ethical principles for medical research involving human subjects. All participants gave and signed the written informed consent form prior to data collection and their right to withdraw at any time of the study was maintained. The results of this study are part of MSc thesis of Ms. Elham Ahmadi (Thesis number: 97789).

\section{Acknowledgments}

We would like to thank all members of the present study group for their ideas, suggestions, participation and support. As well as, the authors wish to thank Kermanshah University of Medical Science for their support.

\section{Author Contributions}

All authors made substantial contributions to conception and design, acquisition of data, or analysis and interpretation of data; took part in drafting the article or revising it critically for important intellectual content; gave final approval of the version to be published; and agree to be accountable for all aspects of the work.

\section{Funding}

RaNCD is part of the PERSIAN national cohort, and we would like to thank Prof. Reza Malekzadeh, the Deputy of Research and Technology at the Ministry of Health and Medical Education of Iran and Director of the PERSIAN cohort, as well as Dr. Hossein Poustchi, the Executive Director of the PERSIAN cohort, for all their support during the design and implementation of the RaNCD cohort. This study was supported by the Ministry of Health and Medical Education of Iran and Kermanshah University of Medical Sciences (grant No. 92472). 


\section{Disclosure}

The authors declare that there is no conflict of interest.

\section{References}

1. Johnson P, Turner L, Carter M, Kelly R, Ewell PJ. Metabolic syndrome prevalence and correlates in a worksite wellness program. Workplace Health Saf. 2015;63(6):245-252. doi:10.1177/ 2165079915576920

2. Rotter I, Kosik-Bogacka D, Dołęgowska B, Safranow K, Lubkowska A, Laszczyńska M. Relationship between the concentrations of heavy metals and bioelements in aging men with metabolic syndrome. Int J Environ Res Public Health. 2015;12(4):3944-3961. doi:10.3390/ijerph120403944

3. Kaur J. A comprehensive review on metabolic syndrome. Cardiol Res Pract. 2014;2014:1-21. doi:10.1155/2014/943162

4. Ebrahimi H, Emamian MH, Khosravi A, et al. Comparison of the accuracy of three diagnostic criteria and estimating the prevalence of metabolic syndrome: a latent class analysis. J Res Med Sci. 2019;24:108

5. Delavari A, Forouzanfar MH, Alikhani S, Sharifian A, Kelishadi R. First nationwide study of the prevalence of the metabolic syndrome and optimal cutoff points of waist circumference in the Middle East: the national survey of risk factors for noncommunicable diseases of Iran. Diabetes Care. 2009;32(6):1092-1097. doi:10.2337/dc08-1800

6. Riccardi G, Giacco R, Rivellese A. Dietary fat, insulin sensitivity and the metabolic syndrome. Clin Nutr. 2004;23(4):447-456. doi:10.1016/j.clnu.2004.02.006

7. Hosseinpour-Niazi S, Mirmiran P, Hosseini-Esfahani F, Azizi F. Is the metabolic syndrome inversely associates with butter, non-hydrogenated-and hydrogenated-vegetable oils consumption: tehran lipid and glucose study. Diabetes Res Clin Pract. 2016;112:20-29. doi:10.1016/j.diabres.2015.11.008

8. Lopez S, Bermudez B, Ortega A, et al. Effects of meals rich in either monounsaturated or saturated fat on lipid concentrations and on insulin secretion and action in subjects with high fasting triglyceride concentrations-. Am J Clin Nutr. 2011;93(3):494-499. doi:10.3945/ ajcn. 110.003251

9. Volk BM, Kunces LJ, Freidenreich DJ, et al. Effects of step-wise increases in dietary carbohydrate on circulating saturated fatty acids and palmitoleic acid in adults with metabolic syndrome. PLoS One. 2014;9(11):e113605. doi:10.1371/journal.pone.0113605

10. Unger AL, Torres-Gonzalez M, Kraft J. Dairy fat consumption and the risk of metabolic syndrome: an examination of the saturated fatty acids in dairy. Nutrients. 2019;11(9):2200. doi:10.3390/nu11092200

11. Organization WH. General meeting of the WHO global coordination mechanism on the prevention and control of noncommunicable diseases: meeting report: International Conference Centre, Geneva, Switzerland, 5-6 November 2018. World Health Organization; 2019.

12. Micha R, Mozaffarian D. Trans fatty acids: effects on metabolic syndrome, heart disease and diabetes. Nat Rev Endocrinol. 2009;5 (6):335. doi:10.1038/nrendo.2009.79

13. Friedrich M, Petzke K, Raederstorff D, Wolfram S, Klaus S. Acute effects of epigallocatechin gallate from green tea on oxidation and tissue incorporation of dietary lipids in mice fed a high-fat diet. Int J Obes. 2012;36(5):735-743. doi:10.1038/ijo.2011.136

14. Singh A, Shenoy S, Sandhu JS. Prevalence of metabolic syndrome and its risk factors among urban sikh population of Amritsar. J Postgrad Med Educ Res. 2015;49(1):18. doi:10.5005/jp-journals $-10028-1137$

15. Hosseini M, Asgary S. Effects of dietary supplementation with ghee, hydrogenated oil, or olive oil on lipid profile and fatty streak formation in rabbits. ARYA Atheroscler. 2012;8(3):119.
16. Sharma H, Zhang X, Dwivedi C. The effect of ghee (clarified butter) on serum lipid levels and microsomal lipid peroxidation. $A Y U$. 2010;31(2):134. doi:10.4103/0974-8520.72361

17. Drehmer M, Pereira MA, Schmidt MI, et al. Total and full-fat, but not low-fat, dairy product intakes are inversely associated with metabolic syndrome in adults. $J$ Nutr. 2016;146(1):81-89. doi:10.3945/ jn.115.220699

18. Pasdar Y, Rezaei M, Darbandi M, et al. Consumption pattern of lipids and the factors affecting their selection among families in Kermanshah (2011). J Kermanshah Univ Med Sci. 2014;18(1):44-52.

19. Poustchi H, Eghtesad S, Kamangar F, et al. Prospective epidemiological research studies in Iran (the PERSIAN Cohort Study): rationale, objectives, and design. Am J Epidemiol. 2018;187(4):647-655. doi:10.1093/aje/kwx314

20. Pasdar Y, Najafi F, Moradinazar M, et al. Cohort profile: Ravansar Non-Communicable Disease cohort study: the first cohort study in a Kurdish population. Int $J$ Epidemiol. 2019;48(3):682-683f. doi:10.1093/ije/dyy296

21. Moradi S, Pasdar Y, Hamzeh B, et al. Comparison of 3 nutritional questionnaires to determine energy intake accuracy in Iranian adults. Clin Nutr Res. 2018;7(3):213-222. doi:10.7762/cnr.2018.7.3.213

22. Azar M, Sarkisian E. Food Composition Table of Iran. Tehran: National Nutrition and Food Research Institute; 1980.

23. Goldberg G, Black A, Jebb S, et al. Critical evaluation of energy intake data using fundamental principles of energy physiology: 1. Derivation of cut-off limits to identify under-recording. Eur J Clin Nutr. 1991;45(12):569-581.

24. Black AE. Critical evaluation of energy intake using the Goldberg cut-off for energy intake: basal metabolic rate. A practical guide to its calculation, use and limitations. Int J Obes. 2000;24(9):1119-1130. doi:10.1038/sj.ijo.0801376

25. Azizi F, Esmaillzadeh A, Mirmiran P. Correlates of under-and over-reporting of energy intake in Tehranians: body mass index and lifestyle-related factors. Asia Pac J Clin Nutr. 2005;14(1):54.

26. Shrivastava AK, Singh HV, Raizada A, et al. Serial measurement of lipid profile and inflammatory markers in patients with acute myocardial infarction. EXCLI J. 2015;14:517. doi:10.17179/excli2015-565

27. Martin SS, Blaha MJ, Elshazly MB, et al. Comparison of a novel method vs the Friedewald equation for estimating low-density lipoprotein cholesterol levels from the standard lipid profile. JAMA. 2013;310(19):2061-2068. doi:10.1001/jama.2013.280532

28. Azizi F, Hadaegh F, Khalili D, et al. Appropriate definition of metabolic syndrome among Iranian adults: report of the Iranian National Committee of Obesity. 2010.

29. Mohammadifard N, Nazem M, Naderi G-A, et al. Effect of hydrogenated, liquid and ghee oils on serum lipids profile. ARYA Atheroscler. 2010;6(1):16.

30. Sserunjogi ML, Abrahamsen RK, Narvhus J. A review paper: current knowledge of ghee and related products. Int Dairy J. 1998;8 (8):677-688. doi:10.1016/S0958-6946(98)00106-X

31. Erfani S, Ghavami M, Shoeibi S, et al. Evaluation of fatty acids and volatile compounds in Iranian ghee by head space-solid phase microextraction coupled with gas chromatography/mass spectroscopy. J Agri Sci Technol. 2020;22(1):147-158.

32. Chaturvedi P, Moseki P, Mazunga K Effects of used sunflower oil and ghee (clarified butter) on lipid profile and antioxidants in SD rats. 2016.

33. Hassanzadeh-Taheri M, Hassanzadeh-Taheri M, Jahani F, Hosseini M. Effects of yoghurt butter oils on rat plasma lipids, haematology and liver histology parameters in a 150-day study. Int J Dairy Technol. 2018;71 (1):140-148. doi:10.1111/1471-0307.12419

34. Lichtenstein AH, Erkkilä AT, Lamarche B, Schwab US, Jalbert SM, Ausman LM. Influence of hydrogenated fat and butter on CVD risk factors: remnant-like particles, glucose and insulin, blood pressure and C-reactive protein. Atherosclerosis. 2003;171(1):97-107. doi:10.1016/j.atherosclerosis.2003.07.005 
35. Mozaffarian D, de Oliveira Otto MC, Lemaitre RN, et al. Transpalmitoleic acid, other dairy fat biomarkers, and incident diabetes: the Multi-Ethnic Study of Atherosclerosis (MESA). Am J Clin Nutr. 2013;97(4):854-861. doi:10.3945/ajcn.112.045468

36. Malinska H, Hüttl M, Oliyarnyk O, Bratova M, Kazdova L. Conjugated linoleic acid reduces visceral and ectopic lipid accumulation and insulin resistance in chronic severe hypertriacylglycerolemia. Nutrition. 2015;31(7-8):1045-1051. doi:10.1016/j.nut.2015.03.011

37. Najafi T, Eghtesadi S, Rezaei M, et al. The effect of Kermanshahi animal oil on serum lipid profile in healthy men. Behbood J. 2010;14 (4).

38. Dugan CE, Aguilar D, Park Y-K, Lee JY, Fernandez ML. Dairy consumption lowers systemic inflammation and liver enzymes in typically low-dairy consumers with clinical characteristics of metabolic syndrome. J Am Coll Nutr. 2016;35(3):255-261. doi:10.1080/ 07315724.2015 .1022637

39. Kurotani K, Karunapema P, Jayaratne K, et al. Circulating odd-chain saturated fatty acids were associated with arteriosclerosis among patients with diabetes, dyslipidemia, or hypertension in Sri Lanka but not Japan. Nutr Res. 2018;50:82-93. doi:10.1016/j.nutres.2017. 12.004

40. Pimpin L, Wu JH, Haskelberg H, Del Gobbo L, Mozaffarian D. Is butter back? A systematic review and meta-analysis of butter consumption and risk of cardiovascular disease, diabetes, and total mortality. PLoS One. 2016;11(6):e0158118. doi:10.1371/journal. pone. 0158118
41. Shah M, Adams-Huet B, Garg A. Effect of high-carbohydrate or high-cis-monounsaturated fat diets on blood pressure: a meta-analysis of intervention trials. Am J Clin Nutr. 2007;85 (5):1251-1256. doi:10.1093/ajcn/85.5.1251

42. Gagliardi ACM, Maranhão RC, De Sousa H, Schaefer EJ, Santos RD. Effects of margarines and butter consumption on lipid profiles, inflammation markers and lipid transfer to HDL particles in free-living subjects with the metabolic syndrome. Eur J Clin Nutr. 2010;64(10):1141-1149. doi:10.1038/ejcn.2010.122

43. Palomäki A, Pohjantähti-Maaroos H, Wallenius M, et al. Effects of dietary cold-pressed turnip rapeseed oil and butter on serum lipids, oxidized LDL and arterial elasticity in men with metabolic syndrome. Lipids Health Dis. 2010;9(1):137. doi:10.1186/1476-511X-9-137

44. Judd JT, Baer DJ, Clevidence BA, et al. Effects of margarine compared with those of butter on blood lipid profiles related to cardiovascular disease risk factors in normolipemic adults fed controlled diets. Am J Clin Nutr. 1998;68(4):768-777. doi:10.1093/ajcn/68.4. 768

45. Gao Z, Yin J, Zhang J, et al. Butyrate improves insulin sensitivity and increases energy expenditure in mice. Diabetes. 2009;58 (7):1509-1517. doi:10.2337/db08-1637

46. Anderson-Vasquez HE, Pérez-Martínez P, Fernández PO, WandenBerghe C. Impact of the consumption of a rich diet in butter and it replacement for a rich diet in extra virgin olive oil on anthropometric, metabolic and lipid profile in postmenopausal women. Nutr Hosp. 2015;31(6):2561-2570. doi:10.3305/nh.2015.31.6.8732

\section{Publish your work in this journal}

Diabetes, Metabolic Syndrome and Obesity: Targets and Therapy is an international, peer-reviewed open-access journal committed to the rapid publication of the latest laboratory and clinical findings in the fields of diabetes, metabolic syndrome and obesity research. Original research, review, case reports, hypothesis formation, expert opinion and commentaries are all considered for publication. The manuscript management system is completely online and includes a very quick and fair peer-review system, which is all easy to use. Visit http://www.dovepress.com/testimonials.php to read real quotes from published authors. 